02

\title{
Возбуждение электронных оболочек быстрых структурных ионов и их вклад в потери энергии ионов
}

\author{
(C) Е.С. Гусаревич
}

Северный (Арктический) фредеральный университет им. М.В. Ломоносова, 163002 Архангельск, Россия

e-mail: gusarevich@gmail.com

(Поступило в Редакцию 28 сентября 2016 г.)

На основе приближения эйконала рассмотрены потери энергии быстрых структурных ионов при столкновениях с атомами. Под структурными ионами понимаются ионы, состоящие из ядра и некоторого количества связанных с ним электронов. Показано, что учет взаимодействия ядра иона с атомом мишени в рамках приближения эйконала может заметно влиять на величину эффективного торможения иона, обусловленного возбуждением его собственных электронных оболочек.

DOI: 10.21883/JTF.2017.06.44523.2048

Исследованиям потерь энергии ионов в веществе с давних пор уделяется много внимания (см., например, [1-4] и приведенные в них ссылки) ввиду практической важности этого вопроса. Обычно в таких процессах в роли снарядов выступают [4-7] структурные ионы, состоящие из ядра и некоторого количества электронов, образующих „шубу“ иона. При нахождении потерь энергии таких ионов, помимо возбуждения частиц среды (мишени), необходимо учитывать также возбуждение электронных оболочек самого иона [5,8-14]. Другими словами, согласно [9-14], эффективное торможение структурных ионов в этом случае можно представить в виде

$$
\kappa=\kappa^{(t)}+\kappa^{(p)}
$$

где $\kappa^{(t)}$ - эффективное торможение иона за счет возбуждения частиц среды, а $\kappa^{(p)}$ торможение иона за счет возбуждения своих собственных электронных оболочек. Учет вклада электронной структуры ионов в эффективное торможение (слагаемое $\kappa^{(p)}$ в $\left.(1)\right)$ рассматривался ранее в рамках первого борновского приближения для случая столкновения ионов с атомарными $[9,11]$ и молекулярными [12] мишенями. В частности, в работе [9] было показано, что учет электронной структуры снаряда может давать заметный вклад (до 10-20\%) в общие потери энергии таких ионов. Непертурбативное рассмотрение потерь энергии быстрых структурных ионов при столкновениях с атомами с учетом одновременных переходов в оболочках снаряда и мишени было проведено в работе [10] в рамках метода сшивки и приближения внезапных возмущений. Обобщение развитой в [10] теории на случай столкновения структурных ионов с молекулами и наночастицами проведено в работах $[13,14]$. Отметим одну общую особенность борновского приближения и приближения внезапных возмущений. В рамках этих двух приближений величина $\kappa^{(p)}$ выражается, хотя и поразному, через энергию взаимодействия структурного иона с мишенью

$$
U=U_{t, n}+U_{t, e},
$$

где $U_{t, n}$ и $U_{t, e}-$ энергии взаимодействия мишени с ядром и электронами иона соответственно. Причем в обоих вышеуказанных приближениях слагаемое $U_{t, n}$ входит в $\kappa^{(p)}$ таким образом [9-14], что оно не влияет на переходы в электронных оболочках иона и может быть опущено при расчете эффективного торможения $\kappa^{(p)}$.

В настоящей работе на основе приближения эйконала, развит непертурбативный метод расчета эффективного торможения $\kappa^{(p)}$ быстрых нерелятивистских структурных ионов при столкновениях с атомами, в рамках которого произведен учет слагаемого $U_{t, n}$ и показано, что в определенных случаях оно может вносить заметный вклад в величину $\kappa^{(p)}$. Особой целью работы является исследование зависимости $\kappa^{(p)}$ от заряда ядра иона. Далее в статье используется атомная система единиц $\hbar=e=m_{e}=1$.

Рассмотрим столкновение быстрого нерелятивистского иона с нейтральным атомом. Пусть $Z$ и $Z_{a}-$ заряды ядер иона и атома соответственно, $N$ - число электронов на оболочках иона $(N<Z), v$ - скорость столкновения, причем $v \gg v_{0}$, где $v_{0}-$ характерная скорость электронов на оболочках иона. Дальнейшее рассмотрение будет проведено в рамках непертурбативного подхода, поэтому никаких ограничений сверху на величину $Z_{a}$ налагаться не будет. Эффективное торможение $\kappa^{(p)}$, определяющее вклад электронной структуры иона в общие потери энергии, может быть рассчитано по формуле [10]

$$
\kappa^{(p)}=\sum_{n}\left(\varepsilon_{n}-\varepsilon_{0}\right) \sigma_{n}
$$

где $\varepsilon_{0}$ и $\varepsilon_{n}-$ энергии начального $|0\rangle$ и конечного $|n\rangle$ состояний иона, $\sigma_{n}-$ сечение неупругого перехода $|0\rangle \rightarrow|n\rangle$. При этом конечное состояние атома мишени не отслеживается и может оказаться произвольным. 
В случае многоэлектронных мишеней $\left(Z_{a} \gg 1\right)$, как отмечено в [10], при расчете эффективного торможения $\kappa^{(p)}$ ион-атомное столкновение можно рассматривать как взаимодействие иона с распределенной в пространстве с некоторой плотностью системой зарядов (ядра атома и его электронов). Для описания распределения электронов в атоме воспользуемся моделью Дирака-Хартри-Фока-Слейтера (ДХФС) [15], согласно которой энергия взаимодействия иона с атомом имеет вид:

$$
U(R, r)=\frac{Z_{a} Z}{R} \phi(R)-\sum_{p=1}^{N} \frac{Z_{a}}{\left|R+r_{p}\right|} \phi\left(\left|R+r_{p}\right|\right),
$$

где $R \equiv\{X, b\}-$ координаты ядра иона относительно атома, $r_{p} \equiv\left\{r_{1}, r_{2}, \ldots, r_{N}\right\}-$ набор координат электронов иона относительно его ядра, $\phi-$ экранирующая функция:

$$
\phi(\rho)=\sum_{i=1}^{3} A_{i} \exp \left(-\alpha_{i} \rho\right),
$$

где $A_{i}$ и $\alpha_{i}-$ постоянные, определенные в [15] для всех атомов с $Z_{a}=1, \ldots, 92$.

Для расчета сечения $\sigma_{n}$ в (3) воспользуемся приближением эйконала и приближением Глаубера [16-18], в результате с учетом (4) получим

$$
\kappa^{(p)}=N \pi \int_{q_{\mathrm{eff}}}^{q_{\max }}\left\langle 0\left|\nabla \Phi \nabla \Phi^{+}\right| 0\right\rangle q d q,
$$

где дифференцирование посредством $\nabla$ производится по координатам любого из $N$ электронов иона,

$$
\nabla \Phi=\frac{1}{2 \pi} \int \exp (-i q b) \exp (i \chi) \nabla \chi d^{2} b
$$

$\chi=\sum_{p=1}^{N} \varphi\left(\left|b+s_{p}\right|\right)-Z \varphi(b), \varphi(b)=\frac{2 Z_{a}}{v} \sum_{i=1}^{3} A_{i} K_{0}\left(\alpha_{i}, b\right)$.

Здесь $s_{p} \equiv\left\{s_{1}, s_{2}, \ldots, s_{N}\right\}$ - проекции координат электронов иона на плоскость параметра удара $b, K_{0}-$ функция Макдональда нулевого порядка, $q_{\max }=2 v$, $q_{\text {eff }}=I_{\text {eff }} / v$, а $I_{\text {eff }}-$ эффективная энергия ионизации снаряда, вводимая по аналогии со „средней“ энергией ионизации атомов в теории торможения Бёте [17], с той лишь разницей, что в нашем случае источником возмущения является не заряженная, а нейтральная частица - атом:

$$
\frac{I_{\mathrm{eff}}}{v}=G^{-1}\left(\frac{1}{N} \sum_{n} N_{n} G\left(\frac{\varepsilon_{n}-\varepsilon_{0}}{v}\right)\right)
$$

где $N_{n}-$ силы осцилляторов [17], $G^{-1}-$ функция, обратная к функции

$$
G(q)=\int_{0}^{q}\left|\int_{0}^{\infty} J_{0}(p b) \exp \{-i Q \varphi(b)\} \varphi(b) b d b\right|^{2} p^{3} d p,
$$

где $Q=Z-N-$ заряд иона, $J_{0}-$ функция Бесселя первого рода нулевого порядка.

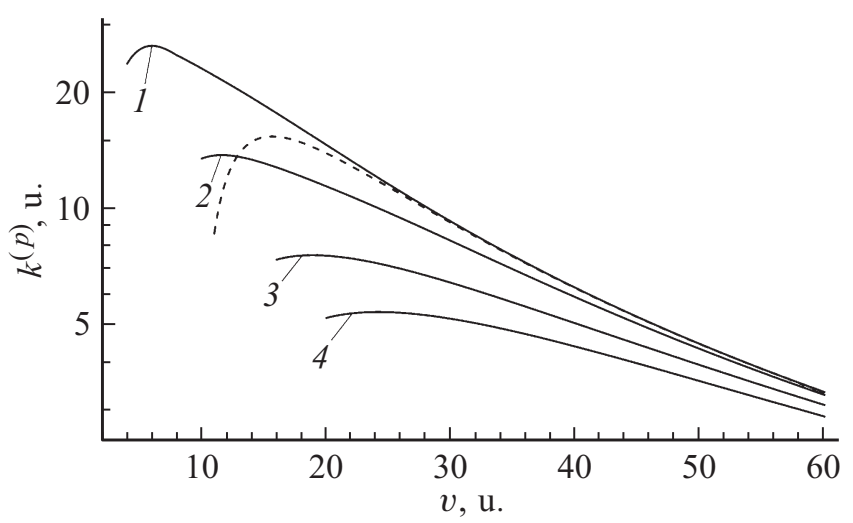

Зависимость эффективного торможения $\kappa^{(p)}$ водородоподобных ионов $\mathrm{He}^{+}, \mathrm{B}^{4+}, \mathrm{O}^{7+}$ и $\mathrm{Ne}^{9+}$ от скорости столкновения $v$ c атомом $\mathrm{Ar}$ (u. - атомные единицы). Сплошная линия расчет по формуле (6), штриховая линия - расчет по аналитической формуле (10). Кривая $1-$ ион $\mathrm{He}^{+}, 2-\mathrm{B}^{4+}$, $3-\mathrm{O}^{7+}, 4-\mathrm{Ne}^{9+}$.

В качестве примера расчета эффективного торможения $\kappa^{(p)}$, мы рассмотрели столкновение с атомом водородоподобного иона. В этом случае в общей формуле (6) $N=1$, а состояния $|n\rangle$ единственного электрона иона описываются водородоподобными волновыми функциями с эффективным зарядом $Z$. При этом $v_{0} \sim Z$, поэтому условие применимости нашего подхода сведется к выполнению неравенства $Z / v \ll 1$. Используя формулу (6) в качестве примера, мы исследовали зависимость эффективного торможения $\kappa^{(p)}$ водородоподобных ионов $\mathrm{He}^{+}$, $\mathrm{B}^{4+}, \mathrm{O}^{7+}$ и $\mathrm{Ne}^{9+}$ на атоме $\mathrm{Ar}\left(Z_{a}=18\right)$ от скорости столкновения. Результаты расчетов представлены на рисунке в виде сплошных линий. Также для сравнения мы привели расчеты $\kappa^{(p)}$ по аналитической формуле (штриховая линия), полученной в работе [10]:

$$
\begin{aligned}
\kappa^{(p)}= & \frac{4 \pi Z_{a}^{2}}{v^{2}} N\left[\ln 2 v-\sum_{i=1}^{3} A_{i}^{2} \ln \left(\alpha_{i} \sqrt{e}\right)\right. \\
& \left.+\sum_{\substack{i, j=1 \\
i \neq j}}^{3} A_{i} A_{j} \frac{\alpha_{j}^{2} \ln \alpha_{j}-\alpha_{i}^{2} \ln \alpha_{i}}{\alpha_{i}^{2}-\alpha_{j}^{2}}+\Delta L_{B}\right],
\end{aligned}
$$

где $\Delta L_{B}$ - поправка Блоха, зависящая лишь от $Z_{a} / v$. Как видно из (10), эта формула не зависит от заряда $Z$ ядра иона, поскольку при ее выводе в [10] использовалось приближение внезапных возмущений, которое, как отмечалось ранее, не чувствительно к взаимодействию ядра иона с мишенью.

Из рисунка видно, что при малых скоростях эффективное торможение $\kappa^{(p)}$, рассчитываемое по формуле (6), оказывается зависящим от заряда ядра иона и существенно отличается от значения, получаемого по формуле (10). Это означает, что при малых $v$ учет взаимодействия ядра иона с мишенью оказывает заметное влияние на значение $\kappa^{(p)}$, рассчитываемое 
в приближении эйконала. Однако с ростом скорости различие в $\kappa^{(p)}$ для разных ионов уменьшается, что свидетельствует об уменьшении влияния вышеуказанного взаимодействия на значение $\kappa^{(p)}$. Анализируя формулу (6), мы установили, что в роли приближенного критерия необходимости учета такого взаимодействия выступает величина $\eta=Z_{a} Z / v$. Если $\eta \gg 1$, то учет взаимодействия ядра иона с мишенью может давать заметный вклад в эффективное торможение $\kappa^{(p)}$. Если же $\eta \leq 1$, то таким взаимодействием можно пренебрегать.

Как видно из рисунка, именно такое поведение наблюдается для ионов $\mathrm{He}^{+}$и $B^{4+}$. При малых скоростях для таких ионов $\eta \gg 1$, поэтому результаты расчетов по формулам (6) и (10) не совпадают друг с другом. Однако при больши́х $v$ параметр $\eta$ становится меньше или порядка единицы, поэтому наши расчетные кривые 1 и 2 (см. рисунок) асимптотически переходят в аналитическое решение (10). В случае же столкновения с атомом ионов $\mathrm{O}^{7+}$ и $\mathrm{Ne}^{9+} \eta \gg 1$ при всех $v$ (так, даже при $v=60: \eta=2,4$ и $\eta=3$ соответственно для $\mathrm{O}^{7+}$ и $\left.\mathrm{Ne}^{9+}\right)$. Поэтому расчеты в рамках приближений внезапных возмущений и эйконала дают заметно отличающиеся друг от друга результаты во всем рассматриваемом диапазоне скоростей.

Представленные в настоящей работе расчеты проводились с использованием библиотек GSL и Cuba на вычислительном кластере САФУ.

Работа выполнена в рамках КГЗ Министерства образования и науки РФ (№ 3.1726.2014/К).

Автор благодарит профессора В.И. Матвеева за обсуждение результатов работы и сделанные полезные замечания.

\section{Список литературы}

[1] Ziegler J.F. // J. Appl. Phys / Rev. Appl. Phys. 1999. V01. 85. P. 1249.

[2] Weaver B.A., Westphal A.J. // Nucl. Instrum. Meth. Phys. Res. B. 2002. Vol. 187. P. 285.

[3] Sigmund P. Particle Penetration and Radiation Effects. General Aspects and Stopping of Swift Point Particles. Springer Series in Solid State Sciences. Berlin: SpringerVerlag, 2006.

[4] Sigmund P. Particle Penetration and Radiation Effects. Vol. 2: Penetration of Atomic and Molecular Ions. Switzerland: Springer, 2014.

[5] Sigmund P.K. // Dan. Vidensk. Selsk. Mat. Fys. Medd. 2006. Vol. 52. P. 557. Special issue on Ion Beam Science: Solved and Unsolved Problems / Ed. by P. Sigmund.

[6] Geissel H., Weick H., Scheidenberger C., Bimbot R., Gardes D. // Nucl. Instrum. Meth. Phys. Res. B. 2002. Vol. 195. P. 3.

[7] Schiwietz G., Grande P.L. // Nucl. Instrum. Meth. Phys. Res. B. 2001. Vol. 175-177. P. 125.

[8] Montanari C.C., Miraglia J.E. // Phys. Rev. A. 2006. Vol. 73. P. 024901.
[9] Cabrera-Trujillo R., Cruz S.A., Oddershede J., Sabin J.R. // Phys. Rev. A. 1997. Vol. 55. P. 2864.

[10] Матвеев В.И., Сидоров Д.Б. // ЖЭТФ. 2007. Т. 132. С. 569.

[11] Tufan M.Ç., Köroğlu A., Gümüş H. // Acta Phys. Pol. A. 2005. Vol. 107. P. 459.

[12] Tufan M.Ç., Kabadayí Ö., Gümüş H. // Radiat. Phys. Chem. 2007. Vol. 76. P. 631.

[13] Матвеев В.И., Гусаревич Е.С., Рябченко С.В., Макаров Д.Н. // Письма в ЖЭТФ. 2008. Т. 88. С. 268.

[14] Матвеев В.И., Гусаревич Е.С., Макаров Д.Н. // ЖЭТФ. 2009. T. 136. C. 843.

[15] Salvat F., Martinez J.D., Mayol R., Parellada J. // Phys. Rev. A. 1987. Vol. 36. P. 467.

[16] Glauber R.J. In Lectures in Theoretical Physics. Vol. I. Ed. by W.E. Brittin. N.Y.: Interscience, 1959. 315 p.

[17] Ландау Л.Д., Лифщщи Е.М. Квантовая механика. М.: Наука, 1989. 768 с.

[18] Joachain C.J., Quigg C. // Rev. Mod. Phys. 1974. Vol. 46. P. 279. 